in underdeveloped countries due to poverty. At the same time, despite the obvious benefits of vaccination, especially in developed countries, there has been a noticeable rise in vaccine hesitancy. With the fall in the number of vaccinated people on a global, national, regional and local level, the risk is growing of epidemics breaking out once again of illness which until recently were in the phase of elimination.

Conclusion Evidence based education, especially the experience and attitudes of all health professionals, as potential educators of the population, is the foundation for successful vaccination. The responsibility of doctors for the implementation of vaccination programmes is great, regardless whether they are directly involved in their realization or not. For the sake of better preparation of future doctors for clinical practice and public health, it is vital to strengthen the quality in undergraduate education about vaccinationin order to overcome the problem of vaccine hesitancy.

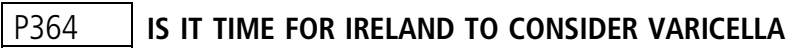 VACCINATION TO THE NATIONAL IMMUNIZATION PROGRAMME?}

1,2 Roy K Philip*, ${ }^{2}$ Trina Dooley, ${ }^{3}$ Janice Murtagh, ${ }^{4}$ Suzanne Cotter. ${ }^{1}$ Graduate entry Medical School, University of Limerick, Limerick, Ireland; ${ }^{2}$ University Hospital Limerick, Limerick, Ireland; ${ }^{3}$ Vaccines division, MSD Ireland Ltd., Dublin, Ireland; ${ }^{4}$ Health Protection Surveillance Centre (HPSC), Dublin, Ireland

\subsection{6/archdischild-2019-epa.710}

Background Varicella (chickenpox) is generally considered a mild illness; however the resultant disease burden is substantial. Uptake of the live-attenuated vaccine to National immunization programmes is variable among European countries. Varicella could pose serious illness and mortality among vulnerable populations such as immunocompromised and those with significant co-morbidities. Varicella vaccine is commercially available in Ireland, however not funded for universal immunization. Since 2012 hospital admissions with varicella is notifiable in Ireland.

Aims To analyse the reporting of hospitalised cases of varicella in Ireland from 2012 to 2018 for 0 to 19 years of age and to propose the potential benefits of inclusion varicella immunization to the National programme.

Methods Surveillance data submitted to health protection surveillance centre (HPSC) from January 2012 to December 2018 from hospitals around all the HSE regions of the country was analysed. Annualised rates for age categories of 0-4 years, 5-9 years, 10-14 years and 15-19 years were determined. Cross verification with hospital inpatient enquiry (HIPE) data was conducted; bed days consumed and length of stay (LOS) were estimated. Results were compared with previously published UK/Irish rates. Mortality was not analysed as part of the study. Approval for analysis of collated data from HPSC and HIPE was obtained.

Results There were 444 hospitalisations for the 0 to 19 years (mean of 63.4 admissions/year). $320 / 44$ (72\%) were in $0-4$ years and 94/444 (21\%) among 5-9 years, together contributing to $93 \%$ of hospitalisations. With a mean LOS of 2.86 days the varicella admissions contributed to 1269.8 bed days (inclusive of general wards, paediatric high dependency unit and paediatric intensive care unit bed utilisation) based on HIPE estimates. An increasing trend of hospitalisation was observed year-on-year for 0-4 and 5-9 years. HPSC reporting was comparable to, however lower than, the active British paediatric surveillance unit (BPSU) study published in 2007 (including Irish data). Our sentinel rate estimate of 133.1/ 100,000 population (range 98.8 -224.7) reflects community burden of varicella.

Conclusions Improving and standardising the varicella surveillance, highlighting the preventable acute hospital bed days due to serious illness from varicella, analysing the disease specific mortality, accurately estimating the disease burden in community including the societal costs and predicting the future implications to rate of herpes zoster among adults and the elderly; all should be factored-in to make a case for the inclusion of varicella to the National immunization programme in Ireland.

\section{P365 PERTUSSIS VACCINATION: SHOULD WE BE DOING SOMETHING DIFFERENT?}

Louise Hendrick* , Mary Ward. Department of Public Health, HSE East, Ireland

\subsection{6/archdischild-2019-epa.711}

Background Pertussis is a highly infectious disease and an important public health concern. The main aim of pertussis vaccination is to reduce the risk of severe pertussis in infants and young children, due to the high disease-related morbidity and mortality in this age group. While the introduction of whole-cell vaccine in the 1940s led to a dramatic decrease in cases and associated fatalities, its higher reactogenicity led to its replacement with acellular vaccines, which contain fewer antigens.(1) In Ireland, despite vaccination coverage rates of 95\% from 2011-2018 average rates from 2014-2018 were more than double those from 2003-2008.(2) Infants under 6 months and adults between 35 and 44 years have been disproportionately affected.(3)

Aim The aim of this review was to assess the effectiveness of wholecellular versus acellular pertussis; to analyse the relevance of recent trends in circulating strains including genetic divergent isolates; to assess the effectiveness of varying the number of antigen components in acellular vaccines and to determine the impact of different vaccination schedules on vaccine efficacy.

Methods A review of the literature was performed across three electronic databases from January 1990 to October 2018, using key search terms. A search of grey literature using the same terms and time period was also conducted.

Conclusion National Vaccination programs have not led to optimal pertussis control. Variations in scheduling or type of vaccine (wholecell or acellular) has not resulted in improved control. The change to acellular vaccination highlighted the relatively short-lived benefit on adaptive immunity and protection and lead to the introduction of booster vaccination doses at a younger age and at adolescence to counter waning immunity. Acellular vaccinations contain between one and five antigenic components. Vaccines which contain between three and five antigens demonstrate higher efficacy than vaccines with lower antigen components. In Denmark and Sweden effectiveness studies have shown some benefit in mono-component and two-antigen vaccines, although factors such as surveillance, diagnosis, variation in case definition and differences in uptake rates make comparison difficult. Mismatches between circulating strains and specific vaccine antigens, particularly pertactin, have been reported, although no evidence of higher virulence has been associated with these isolates. 
Whilst several variations in scheduling exist no superiority was demonstrated with any one approach.

The results of this review do not indicate the benefit of a change in the scheduling or vaccine component of pertussis vaccines currently used in the Irish childhood vaccination programme.

\section{P366 THE INTEREST OF ANTI HEPATITIS B VACCINATION AT BIRTH}

Yakoubou Annatou*, Tchiakpè Nicole. Faculté des Sciences de la Santé; Université D'abomey Calavi, Cotonou, Benin

\subsection{6/archdischild-2019-epa.712}

In Benin, anti hepatitis $\mathrm{B}$ vaccination begins at six weeks of age under the EPI, while WHO recommends a dose at birth to prevent perinatal transmission, which is responsible for the onset of chronic infections.

Objective To compare vaccine seroconversion against hepatitis B between 9-month-old children who had received or not a dose of anti hepatitis B vaccine at birth in two health units at Cotonou.

Methods This was a cross-sectional study. we included 9month-old children and their mothers received in vaccination at CHU-MEL (where vaccination at birth was automatically offered) and the primary health care of Cotonou I (where only the EPI was offered). The study occurred from April to June 2017. The socio-demographic and immunization data (immunization status, anti HBS, HBS Ag) were studied. They had been processed and analysed with Excel 10 and SPSS 21 software. Pearson's student and correlation tests were used for comparisons and the significance threshold was $5 \%$.

Results A total of 128 mother-child couples were recruited; Half of the children were vaccinated according to the 4-dose regimen and the other, according to the 3-dose regimen. The incidence of hepatitis B was $9.38 \%$ for mothers $(n=12)$ and $1.62 \%$ for children $(n=2)$. The average antibody title was $617 \mathrm{IU} / \mathrm{l}$ in children at 4 doses versus $395 \mathrm{UI} / \mathrm{L}$ in 3 -dose patients. This difference was statistically significant $(p=$ 0.023).

Conclusion The 4-dose vaccine regimen, one at birth, provides a better immune response. The inclusion of vaccination against hepatitis B at birth in the EPI is indispensable.

\section{P367 TO SWITCH OR NOT TO SWITCH: THE BENEFIT OF QUADRIVALENT INFLUENZA VACCINE TO THE IRISH PAEDIATRIC POPULATION}

Julie Arnott* ${ }^{*}$ Annalisa Quattrocchi, Lisa Domegan, Meadhbh Hunt, Paula Flanagan, Joan O Donnell. HSE Health Protection Surveillance Centre, Dublin, Ireland

\subsection{6/archdischild-2019-epa.713}

Background Until recently, trivalent influenza vaccines (TIV) have contained one influenza B virus, recommended annually by the WHO vaccine selection committee. Quadrivalent Influenza Vaccines (QIV) add protection against a second B lineage; preventing the threat of a vaccine mismatched season, which often occurs in the Northern Hemisphere, leading to reduced vaccine effectiveness (VE) and increased influenza morbidity and mortality. Young children have one of the highest clinical burdens in Ireland, with the highest age-specific rate for influenza cases admitted to critical care units generally in children aged 0-4 years. Additionally, school-aged children are a major source of community transmission.

Aim To assess the benefit of a QIV in the Irish paediatric population.

Methods A literature review was conducted comparing QIV to TIV, focusing on VE and cost-effectiveness (CE). The VE of live-attenuated influenza vaccine (LAIV) compared to inactivated influenza vaccine (IIV) in children was also studied. The Cochrane database of systematic reviews, the Cochrane Central Register of Controlled Trials, PubMed, the Lancet, and publications from the European projects I-MOVE/I-MOVE+ and VENICE were searched for publications between 20092018.

Results Recent influenza seasons suggest a higher VE of QIV compared to TIV. Studies have shown that QIV is as effective as TIV for the strains included in both; however QIV has superior immunogenicity for the additional B strain when there is a mismatch season. These results are also reflected in pre-licensing studies of the immunogenicity of QIVs that are now approved.

LAIV has been recommended due to higher VE against influenza B strains and the ease and acceptability of the intranasal vaccination compared to the injectable IIV.

QIV are more expensive than TIV, however CE analyses indicate that QIV delivers substantial savings in terms of preventing direct healthcare costs through reductions in infection numbers, hospitalisations and deaths; resulting in qualityadjusted life years gained. There are also substantial societal benefits through indirect savings in productivity (preventing employee/caregiver absences). Rolling out QIV to children was the most cost-effective vaccination strategy in the UK (aged 211 years) and in European countries (4-16 years) partaking in the I-MOVE+ project, with the exception of Portugal.

Conclusion QIV would stabilise the VE across influenza seasons; eliminating the uncertainty of predicting the influenza B lineage, ultimately increasing public confidence in the vaccine, resulting in increased vaccine uptake. Broader protection in the paediatric population would directly reduce influenza transmission and indirectly protect vulnerable populations in the community.

\section{P368 FREQUENCY OF DIARRHEA AND PNEUMONIA IN VACCINATED AND UNVACCINATED CHILDREN UNDER 5 YEARS OF AGE: A SINGLE CENTER STUDY}

${ }^{1}$ Shireen Qassim Bham*, ${ }^{1}$ Farhan Saeed, ${ }^{2}$ Muhammad Athar Khan, ${ }^{3}$ Rashid Naseem Khan, ${ }^{4}$ Fatima Siraj, ${ }^{4}$ Syeda Sara Afsar, ${ }^{4}$ Tayyab Mehmood. ${ }^{1}$ Department of Pediatrics; ${ }^{2}$ Department of Community Medicine; ${ }^{3}$ Department of Medicine; ${ }^{4}$ Medical Students

\subsection{6/archdischild-2019-epa.714}

Background Pneumonia and diarrhea remain the leading infectious causes of mortality and morbidity in children under 5 years of age. ${ }^{1}$ According to the study carried out by WHO in 2017 the coverage of rotavirus is $23 \%$ while that of pneumococcal vaccine is $43 \%$ which accounts for serious health issues in children. ${ }^{2}$ An estimated 1 in 40 infants experience a severe episode of rotavirus gastroenteritis annually in Pakistan. ${ }^{3}$

Objective The objective of this study was to determine the frequency of diarrhea and pneumonia in vaccinated and unvaccinated children less than 5 years of age: A single center study 